\title{
THE MODE OF ACTION OF PENICILLIN AND ITS POSSIBLE SIGNIFICANCE FOR THERAPY
}

\author{
J. T. PARK \\ Department of Microbiology, Tufts University School of Medicine, Boston, Mass., U.S.A.
}

THE objectives of my discussion are to review briefly what is known of the mode of action of the penicillins, then to consider in more detail our recent work on biosynthesis of cell wall mucopeptide and the effect of penicillin thereon, and finally, to consider possible problems in the clinical use of penicillins suggested by our knowledge of the mode of action. The principal properties of penicillin action have been described from 7 to 20 years ago, and for this discussion it is assumed that the new penicillins all have basically the same mode of action.

First, let me mention several well-known effects of the interaction of penicillin with sensitive cells. The immediate consequences of this interaction are that some penicillin binds irreversibly to the cells, and the synthesis of cell wall mucopeptide is inhibitedsometimes with the concomitant accumulation of precursors as in the staphylococcus. This has focused attention on mucopeptide biosynthesis although it is not yet proven that penicillin directly inhibits a reaction in this pathway. The steps in the synthesis of mucopeptide precursors by the staphylococcus are believed to be as shown in Figure 1. The enzymes which add the amino acids to UDP -acetyl muramic acid in this stepwise manner have been shown to be present in the staphylococcus by Ito and Strominger (1963). Since the uridine nucleotide containing the pentapeptide (UDP-muramyl peptide) is the principal one to accumulate in penicillin-inhibited staphylococci it has been postulated that penicillin inhibits a step in mucopeptide synthesis subsequent to formation of the pentapeptide such as a polymerization reaction utilizing UDP-muramyl peptide.

The polymerization reaction may be visualized as shown in Figure 2. A polysaccharide chain is formed by alternate transfer of acetyl glucosamine and acetyl muramyl peptide from the uridine-bound precursors to the end of the chain. In the staphylococcus, another peptide composed of about five glycine residues is attached to the $\xi$-amino group of lysine and the amino end of the glycine peptide is presumed to be linked to glutamic acid or alanine to form cross links between the repeating units of the chain or neighbouring chains.

Fig. 1

Steps in the Biosynthesis of a Precursor of Staphylococcal mucopeptide.

UDP-acetyl muramyl-L-alanyl-

-D-glutamic acid<smiles>CCC</smiles>

UDP-acetyl muramyl-L-alanine

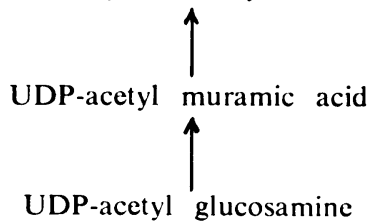

UDP-acetyl muramyl-L-alanyl-

-D-glutamyl-L-lysine

D-alanyl-D-alanine

UDP-acetyl muramyl-L-alanyl

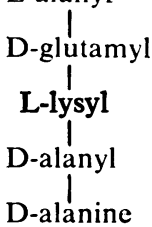


Fig. 2.

Possible polymerization reaction utilizing UDP-muramyl peptide for the formation of Staphylococcal mucopeptide.

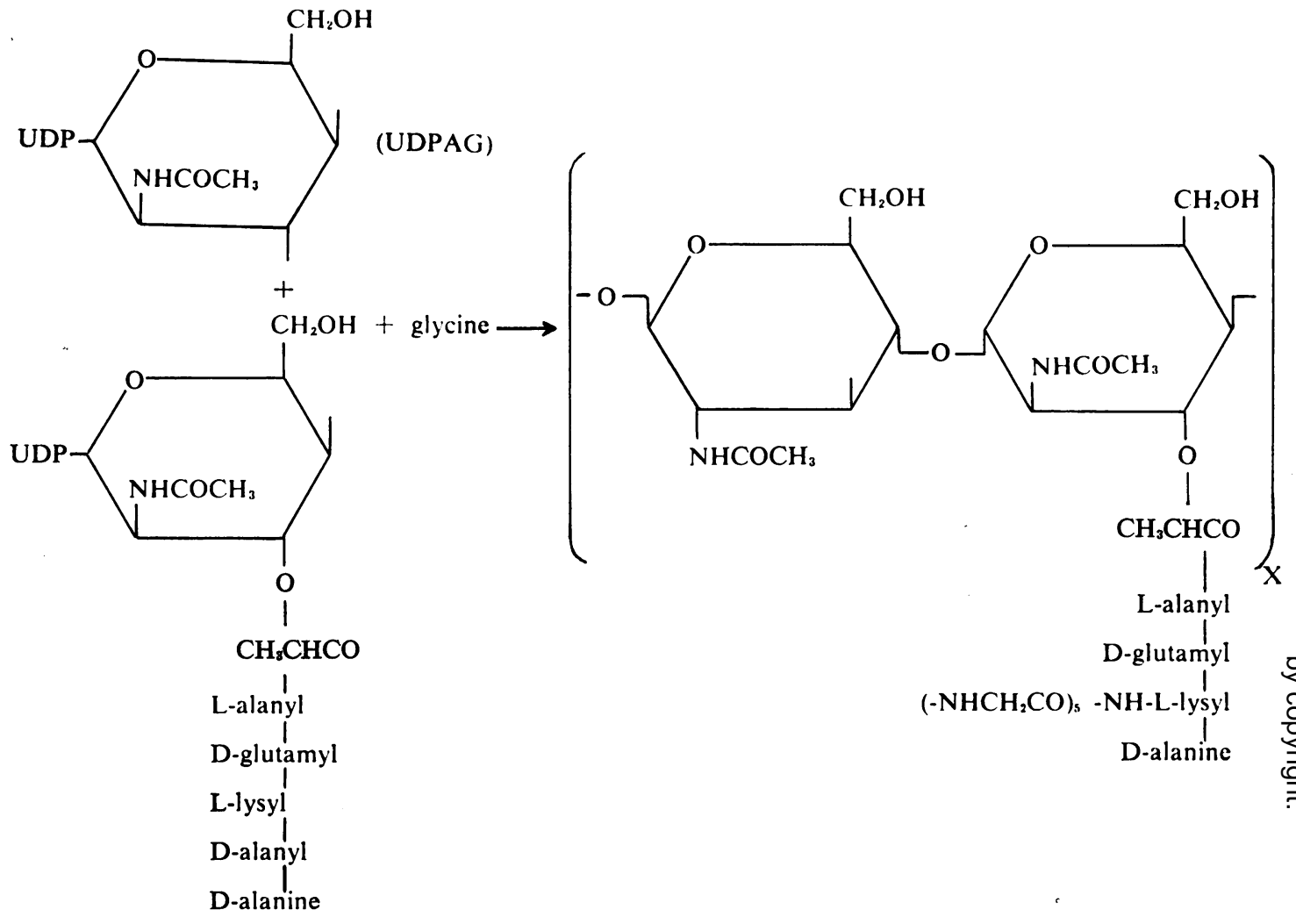

(UDP-muramyl peptide)

This specific type of heteropolymer which contains glucosamine, muramic acid, L- and D-alanine, D-glutamic acid, and a dibasic amino acid such as lysine or diaminopimelic acid has been termed mucopeptide. Mucopeptide is believed to be an essential wall constituent needed to preserve the cellular integrity of all bacteria; and of the actinomyces, rickettsia, and organisms of the psittacosis-lymphogranuloma group as well. These organisms are the ones which are sensitive to penicillin though admittedly many of them are not sensitive to therapeutic levels of benzyl penicillin. Because penicillin action and mucopeptide formation seem related, an understanding of the final stages of mucopeptide biosynthesis and the effect of penicillin on these final stages is of great interest.
The final polymerization reaction has been investigated recently by Chatterjee and myself and by Meadow, Thompson and Strominger (1964). In our studies (Chatterjee and Park, 1964) most experiments were carried out with $\delta$ two radioactive substrates, $\mathbf{H}^{3}$-glycine and UDP-muramyl peptide containing $\mathrm{C}^{14}$-lysine. It was found that UDP-muramyl peptide was utilized by broken cells but not by intact cells, and the activity proved to be associated with particles of membrane. In addition to membrane particles, magnesium, ATP, and ribosomes stimulated the utilization of UDP. muramyl peptide. Glycine was also incorporated by the membrane particles and all of 6 the above materials plus $\mathrm{KCl}$ and at least two unidentified soluble constituents of the cell were needed. 
A study of the substrate requirements for the reaction showed that UDP-acetyl glucosamine was required for both muramyl peptide and glycine incorporation and that the complete UDP-muramyl peptide was required for incorporation of glycine: neither UDP-muramic acid nor UDP-muramyl alanyl-glutamyl-lysine would substitute for UDP-muramyl peptide. Thus the reaction studied was highly specific. These experiments indicate that UDP-muramyl peptide is utilized by cells, and therefore is a natural precursor.

How does penicillin affect this reaction? When it, vancomycin, and bacitracin (two other antibiotics which appear to act in a similar but not identical manner) were tested in the cell-free system it was found that they did not inhibit the reaction. However, cells treated during growth with one of these antibiotics for 20 minutes yielded membrane particles of greatly reduced activity. The same type of result was obtained in studies with intact cells. Cells treated in buffer with penicillin and then, after the removal of unbound penicillin, tested for their ability to synthesize mucopeptide had essentially normal activity but if the cells were treated during growth much of their mucopeptide synthesizing activity was lost. Cells treated for a short time with penicillin during growth were still viable, however. During the recovery of inhibited cells in growth medium, the ability to synthesize mucopeptide is restored within 20 minutes. If tetracycline or other inhibitor of protein synthesis is present in the growth medium recovery does not occur (Park, Griffith and Stevenson-unpublished). This suggests that recovery requires synthesis of a new protein to replace that damaged by penicillin. This new evidence may eventually help us understand why penicillin acts only on growing cells.

Before considering what all this may have to do with therapy, it should be pointed out that the recent evidence raises some doubt as to whether penicillin is acting directly on the mucopeptide pathway, since penicillin does not inhibit the synthesis in a cell-free system. The second reason for doubt is that vancomycin and bacitracin, which show no cross resistance with penicillin, appear to affect the same pathway. It is possible that all are acting indirectly. However, the peculiar dependence on growth conditions to demonstrate the biological and biochemical effects of penicillin leads us to believe that penicillin must interfere directly with the synthetic reaction.

\section{Problems which may arise during clinical use of penicillin}

Regardless of the exact mode of action of penicillin at the molecular level, it is well known that it acts only on growing cells, and that sensitive cells are often converted to spheroplasts which are insensitive to penicillin. Depending on the environment the spheroplasts will lyse or they may survive and actually grow or at least survive and be able to revert to the bacillary form after removal from penicillin. These facts raise three basic problems which, so far as I know, have not been thoroughly evaluated in clinical practice. Since, on theoretical grounds these problems must exist, presumably they affect the outcome of therapy to some extent in all cases and in a crucial way in an unknown though small percentage of cases. For this reason the following problems should not be ignored.

1. The problem of spheroplasts. How great is the danger that spheroplasts will survive the course of therapy and then revert to the bacillary form to cause relapse? It has been suggested in the past that spheroplasts may be able to survive for long periods in the kidney. Is this a problem? If so, should a second course of therapy to destroy the spheroplasts be considered? Is there ever a situation in which penicillin cures a disease only to leave the patient suffering from a lesser complaint caused by the survival and multiplication of the spheroplasts?

2. The problem of lysis of spheroplasts. Conceivably the sudden lysis of the invading organisms by penicillin could release a fatal dose of toxins. Are there acute diseases in which a bacteriostatic drug might be preferable for this reason? Does the oral administration of penicillins in high dosage ever cause the sudden lysis of the Gram-negative intestinal flora with the release of endotoxins which nullify the treatment?

3. The problem of penicillin acting only on growing cells. The observation of Jawetz and Gunnison (1952) that the bactericidal action of penicillin can be antagonized by bacteriostatic drugs such as chloramphenicol or tetracycline can be explained on theoretical grounds. 
The bactericidal action of penicillin depends upon bacterial growth and any condition which is unfavourable for growth is likely to be antagonistic.

What is the best way to use penicillin in view of these problems? It would seem that answers to all of the questions posed above are needed before the most prudent use of penicillin can be achieved.

\section{REFERENCES}

Chatterjee, A. N., and Park, J. T. (1964), Proc. na丣 Acad. Sci. (Wash.), 51, 9.

Ito, E. and Strominger, J. L. (1963), J. biol. Chem 237, 2689.

JAWETZ, E., and GunNison, J. B. (1952), Antibiot and Chemother., 2, 243.

Meadow, P. M., Thompson, J. S., and Strominger J. L. (1964), Biochem. biophys. Res. Commur 14, 382 .

\title{
INTERPRETATION OF ASSAY VALUES
}

\author{
E. T. KNUDSEN
}

Beecham Research Laboratories, Brentford, Middlesex.

THE appearance of new antibiotics in everincreasing numbers demands a critical appraisal of the data usually presented relating to their therapeutic properties. Generally speaking, the common investigations undertaken to assess antibiotic potential are liable to a number of sources of error.

The investigations fall into five main groups:

1. In vitro activity studies (broth or serum).

2. Serum concentration determinations and "serum" antibacterial activity studies.

3. "Tissue" concentrations (animal or man).

4. Chemotherapeutic assessment in animals.

5. Clinical trials in man.

In vitro activity studies determine the minimum inhibitory concentration (M.I.C.) of an antibiotic for a given organism-the figure so obtained depends, among other factors, upon whether the determination is carried out in broth or in serum. The origin of the serum (whether human or animal) will influence the result as well as the concentration of the serum used in the determination $(40 \%, 60 \%, 80 \%$, $95 \%$ ).

Table I shows the M.I.C.s of a penicillin for an organism obtained in broth culture and in $95 \%$ human serum.

TABLE I

Minimum Inhibitory Concentrations ( $\mu \mathrm{g} . / \mathrm{ml})$

$\begin{array}{llr}\text { Broth Culture } & = & 1.0 \\ 95 \% \text { Human Serum } & = & 4.0 \\ \text { Loss of Activity } & = & 75 \%\end{array}$

Total $\quad$ Protein-bound fraction $=75 \%$ Penicillin $\left\{\begin{array}{l}\text { Pree (active) fraction }=25 \% \\ \text { F }\end{array}\right.$
It will be seen that in the presence of $95 \%$ human serum this penicillin loses some $75 \%$ of its activity compared with the figure obtained from broth culture. This los $\$$ of activity is due to the reversible binding of a proportion of the penicillin to the serund proteins. Bound penicillin is inactive and in th@ example shown in Table $I$, only approxi通 ately $25 \%$ of the penicillin remains in the free (active) form.

Many antibiotics lose activity in the prese of serum and in the case of penicillins, there good correlation between the loss of activity in $95 \%$ human serum and the degree of protein binding.

The minimum inhibitory concentrations of an antibiotic generally published for a range of organisms are those obtained from broth culture and there is often inadequate informa tion about the extent or effect of protein binding.

Consideration of the minimum inhibitory concentrations of a penicillin obtained from broth culture in conjunction with serum concentrations following a given dosage can be్ very misleading; for the methods usually employed to determine the serum concentrations of a penicillin give only total serum level, thap is to say, the figure so obtained refers to the sum of the free and protein-bound fractions $N$

Estimations of total serum levels of the penicillin are shown in Table II and Figure 1요 If the total serum concentration curve (Fig. 1) is considered in relation to the M.I.C. for the organism obtained in broth culture, it wiff be seen that the serum level exceeds thes M.I.C. for almost 4 hours; but if the M.I.C. obtained in the presence of $95 \%$ of 